\section{Response to: 'The effects of a brace for patellofemoral osteoarthritis targeting knee pain and bone marrow lesions were overestimated or not?' by Zeng et al}

As we noted in our paper reporting results of the BRACE trial, ${ }^{1}$ a trial of patellar bracing in patellofemoral osteoarthritis (OA), we did not use a placebo control because all such feasible controls recapitulated at least one mechanism of action of the patellar brace, and we were interested in examining whether any action of the brace might be therapeutic. Our means of demonstrating the effect of the brace was to examine compartmentspecific bone marrow lesion (BML) change on MRI in addition to pain reduction. We showed that patellofemoral BMLs shrank in the active group versus control.

As for the concern about physical activity being less in the active group during the trial, we did not assess activity during the trial, and it is unclear what effect greater or less physical activity might have. We note that BMLs in the tibiofemoral compartment did not change in the groups, and to the extent that these lesions are affected by trauma from activity, this suggests that activity did not differ between groups. It is possible, as Zeng et al suggest, ${ }^{2}$ that specific activities differed in the two treatment groups during the trial. One reason the brace group had less knee pain might be that the placement of the brace discouraged participants from carrying out painful knee flexion activities.

We did not collect data on the contralateral knee, as we chose to examine and focus on the more symptomatic knee.

Participants in the study were not undergoing active physical therapy, and had to be on stable medication treatment before and during the trial. A qualitative change in medication would have constituted a protocol violation, and we ensured that no such change occurred. We did not track the quantity of analgesics used, but studies have shown that analgesic use change tracks pain change closely in OA trials. ${ }^{3}{ }^{4}$

As for other outcomes, function (both based on Knee Injury and Osteoarthritis Outcome Score (KOOS) and other measures) also showed statistically significant improvement in the brace group compared with the control. We followed all subjects for an additional 12 weeks in an open-label extension of the trial, and will be reporting these results soon.

Zeng et al are correct that patellofemoral was not fully defined in the abstract; it is in the main text. Figure 1 should have the colours reversed. We appreciate the correction.

Michael Callaghan, ${ }^{1}$ Matthew Parkes, ${ }^{1}$ David T Felson ${ }^{1,2}$

${ }^{1}$ University of Manchester, Manchester, UK

${ }^{2}$ Boston University School of Medicine, Boston, Massachusetts, USA

Correspondence to Professor David T Felson, Boston University School of Medicine, A203, 80 E. Concord Street, Boston, MA 2118, USA; dfelson@bu.edu

Twitter Follow Matthew Parkes at @mattyjparkes

Competing interests None declared.

Provenance and peer review Commissioned; internally peer reviewed.

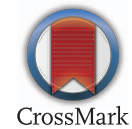

To cite Callaghan M, Parkes M, Felson DT. Ann Rheum Dis 2015;74:e52.

Received 15 April 2015

Accepted 19 April 2015

Published Online First 6 May 2015

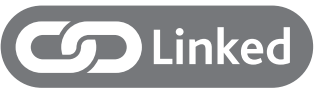

http://dx.doi.org/10.1136/annrheumdis-2015-207673

Ann Rheum Dis 2015;74:e52. doi:10.1136/annrheumdis-2015-207681

\section{REFERENCES}

1 Callaghan MJ, Parkes MJ, Hutchinson CE, et al. A randomised trial of a brace for patellofemoral osteoarthritis targeting knee pain and bone marrow lesions. Ann Rheum Dis 2015. Published Online First 16 Jan 2015. doi: 10.1136/ annrheumdis-2014-206376

2 Zeng C, Gao S-guang, Lei G-hua. Ann Rheum Dis 2015. Published Online First 9 Apr2015. doi:10.1136/annrheumdis-2015-207673

3 Bennell KL, Bowles KA, Payne C, et al. Lateral wedge insoles for medial knee osteoarthritis: 12 month randomised controlled trial. BMJ 2011;342:d2912.

4 Baker $\mathrm{K}$, Goggins J, Xie H, et al. A randomized crossover trial of a wedged insole for treatment of knee osteoarthritis. Arthritis Rheum 2007:56:1198-203. 